\title{
PDGF mediates pulmonary arterial smooth muscle cell proliferation and migration by regulating NFATc2
}

\author{
FANG-YUN ZHAO ${ }^{1}$, SHUANG-LAN XU² ${ }^{2}$ CHUN-FANG ZHANG ${ }^{3}$, JIE LIU ${ }^{2}$, \\ YUE ZHANG $^{1}$, JIAO YANG ${ }^{4}$ and XI-QIAN XING ${ }^{2}$
}

\begin{abstract}
${ }^{1}$ Department of Pharmacy, Yan'an Hospital Affiliated to Kunming Medical University, Kunming, Yunnan 650051; ${ }^{2}$ Department of Respiratory Medicine, The Fourth Affiliated Hospital of Kunming Medical University, The Second People's Hospital of Yunnan Province, Kunming, Yunnan 650021; ${ }^{3}$ Department of Geriatrics, The Affiliated Hospital of Jiangsu University, Zhenjiang, Jiangsu 212000; ${ }^{4}$ First Department of Respiratory Medicine, First Affiliated Hospital of Kunming Medical University, Kunming, Yunnan 650032, P.R. China
\end{abstract}

Received May 7, 2020; Accepted October 13, 2020

DOI: $10.3892 / \mathrm{mmr} .2020 .11677$

\begin{abstract}
The reconstruction of pulmonary vascular structure caused by the proliferation and migration of pulmonary arterial smooth muscle cells (PASMCs) is the central link in the formation of pulmonary arterial hypertension (PAH). Platelet-derived growth factor (PDGF) can regulate the proliferation and migration of PASMCs. At the same time, nuclear factor of activated T cells (NFATs) plays an important role in the development of PAH. To the best of our knowledge, there are no reports yet regarding whether PDGF regulates NFATc2 to increase the proliferation of PASMCs. The present study aimed to investigate whether PDGF affects the proliferation and migration of PASMCs by regulating NFAT, and to study the pathogenesis of PAH. PASMCs were treated with recombinant PDGF; Cell Counting Kit-8 and clone formation experiments showed that PDGF enhanced the cell viability and proliferation of PASMCs. Cell cycle distribution and molecular markers related to cell proliferation (cyclin D1, CDK4 and Proliferating Cell Nuclear Antigen) were detected by flow
\end{abstract}

Correspondence to: Dr Jiao Yang, First Department of Respiratory Medicine, First Affiliated Hospital of Kunming Medical University, 295 Xichang Road, Kunming, Yunnan 650032, P.R. China

E-mail: yangjiaokmu@yahoo.com

Dr Xi-Qian Xing, Department of Respiratory Medicine, The Fourth Affiliated Hospital of Kunming Medical University, The Second People's Hospital of Yunnan Province, 176 Qingnian Road, Kunming, Yunnan 650021, P.R. China

E-mail: xingxiqiankm@163.com

Abbreviations: PASMCs, pulmonary arterial smooth muscle cells; PAH, pulmonary arterial hypertension; PDGF, plateletderived growth factor; NFAT, nuclear factor of activated T cells; CsA, cyclosporin A

Key words: NFAT, PDGF, pulmonary arterial hypertension, pulmonary arterial smooth muscle cells cytometry, and the results indicated that PDGF promoted the division of PAMSCs. The scratch migration and Transwell migration assays showed that the migratory ability of PASMCs was enhanced following PDGF treatment. Changes in NFATs (NFATc1-5) after PDGF treatment were evaluated by reverse transcription-quantitative PCR and western blotting; NFATc2 showed the most significant results. Finally, PDGF-treated cells were treated with an NFAT pathway inhibitor, cyclosporin A, or a small interfering RNA targeting NFATc2, and changes in cell proliferation and migration were evaluated to assess the role of NFATc2 in PDGF-induced cell proliferation and migration. In conclusion, PDGF may regulate PASMC proliferation and migration by regulating the expression of NFAT, further leading to the occurrence of PAH. It is proposed that NFATc2 could be used as a potential target for PAH treatment.

\section{Introduction}

Pulmonary arterial hypertension (PAH) is a relatively rare, debilitating and deadly lung disease diagnosed as a hemodynamic disease through a cardiac catheter (1). There are a number of causes of pulmonary hypertension, such as collagen disease, human immunodeficiency virus infection and anorexia (2). PAH is characterized by continuous vasoconstriction, rapid remodeling of small blood vessels, vascular proliferation, and the aberrant growth of pulmonary arterial smooth muscle cells (PASMCs), leading to a gradual increase in pulmonary vascular resistance, and ultimately to right ventricular failure and death (3). The migration and proliferation of PASMCs are the main pathological bases of pulmonary arterial remodeling and pulmonary hypertension $(3,4)$. Inhibition of abnormal proliferation and migration of PASMCs can effectively reverse pulmonary artery remodeling, thereby reducing pulmonary circulation resistance. Therefore, elucidating the underlying mechanism of PASMC proliferation is expected to provide novel targets for PAH treatment (5). Activated platelet-derived growth factor (PDGF) is composed of polypeptides (A and B chains), forming homo- or heterodimers and stimulating the surface receptors of A and B cells (6). Abnormal PDGF signaling may 
lead to atherosclerosis and aberrant remodeling of peripheral blood vessel-related vessels (7). According to the literature, the expression of PDGF and its receptor is increased in PAH PASMCs $(8,9)$. PDGF has been demonstrated to be a powerful mitogen that stimulates excessive cell proliferation, and has the ability to stimulate the excessive division and proliferation of PASMCs both in vitro and in vivo (10).

Nuclear factor of activated T cells (NFATs) were first discovered in $\mathrm{T}$ cells as their main activator. The primary function of NFATs is to increase the transcription of inflammatory mediators and activate both T and B cells (11). Although inflammation is known to play a role in PAH, it has not yet been adequately studied. According to related articles, an increase in inflammatory mediators has been found in the serum of patients with $\mathrm{PAH}$ and in remodeled PA, and there is a clinical link between PAH and autoimmune diseases such as scleroderma $(12,13)$. NFAT expression or function has been described in several types of non-lymphocytes, including mast, endothelial and neuronal cells (14). The PAH animal model also showed NFAT expression in PASMCs (15). Bonnet et al (16) suggested that the $\mathrm{Ca}^{2+}$-NFAT signaling pathway in patients with idiopathic pulmonary hypertension is activated, which manifests as an increase in $\mathrm{Ca}^{2+}$ and activation of calcineurin $(\mathrm{CaN})$, thereby promoting the phosphorylation of NFAT and its transposition. Once inside the nucleus, NFAT combines with DNA sequences to promote transcription and cell proliferation. Pulmonary arterioles in normal subjects and those with secondary pulmonary hypertension have low expression of NFAT1, whereas those with idiopathic pulmonary hypertension have a high expression of NFAT1 (17). PAH is associated with abnormal expression and activity of NFAT (17). Studies have demonstrated that the expression of NFAT3 protein in the pulmonary arteries of PAH animal models is increased, and serotonin 5-HT2A receptor antagonists can downregulate the expression of NFAT protein, indicating that 5-HT2A receptors may be involved in the process of NFAT3 upregulation during PAH (18-20). A possible explanation is that activation of the 5-HT2A receptors leads to an increase in intracellular $\mathrm{Ca}^{2+}$, which promotes NFAT activation and translocation into the nucleus to combine with gene sequences that promote proliferation and inhibit apoptosis, resulting in cell proliferation and eventually pulmonary artery refactoring.

PDGF reportedly regulates NFAT (21). However, how PDGF and NFAT are related to the pathogenesis of PAH remains unclear. Therefore, the present study aimed to explore the interaction between PDGF and NAFT in the pathogenesis of PAH.

\section{Materials and methods}

Subculture and identification of PASMCs. PASMCs were cultured following type II collagenase digestion (22). A Sprague-Dawley rat (aged 3 months and weighing $250 \mathrm{~g}$ ) was anesthetized with intraperitoneal sodium pentobarbital injection. The anesthetized rat was fixed on the operating table, the chest cavity was exposed, and the distal pulmonary artery was harvested under a stereomicroscope with microsurgical scissors. After removing the endothelial tissues, the blood vessels were cut into small pieces with scissors and placed in type II collagenase to digest flocculent material. The digestion was terminated with 20\% DMEM (Gibco; Thermo Fisher Scientific, Inc.) in high-sugar culture solution and then centrifuged at $200 \mathrm{x} \mathrm{g}$ for $5 \mathrm{~min}$ at $4^{\circ} \mathrm{C}$. The lower layer of the precipitate was removed, resuspended in the DMEM solution, and cultured in a flask. The fluid was changed the next day, and identification was performed 1 week later. Primary cultured cells were subcultured in flasks. PASMCs with good growth from the second to sixth passages were used for subsequent experiments. Anti- $\alpha$-actin immunohistochemical staining was used to identify PASMCs. PASMCs treated with PDGF were the PDGF group; PASMCs treated with PBS were the Vehicle group; PASMCs without any treatment were the Blank group. Cyclosporin A (CsA, C-3662) was purchased from Sigma-Aldrich (Merck KGaA). The PASMCs were treated with $0.5 \mu \mathrm{g} / \mathrm{ml} \mathrm{CsA}$ for $48 \mathrm{~h}$ in the Colony formation and Transwell experiments. The present study was approved by the Ethics Committee of The Fourth Affiliated Hospital of Kunming Medical University, The Second People's Hospital of Yunnan Province (Kunming, China).

Cell viability and proliferation. A Cell Counting Kit- 8 (CCK-8) assay was used to detect PASMC viability. PASMCs were treated with different concentrations $(0,10,20,50,80$, 100 and $200 \mathrm{ng} / \mathrm{ml}$ ) of PDGF (PeproTech Inc.) from day 1 to day 3 of the cell culture at $37^{\circ} \mathrm{C}$ with $5 \% \mathrm{CO}_{2}$, cells treated with $0 \mathrm{ng} / \mathrm{ml}$ PDGF served as Control. Cells were seeded onto 96-well plates in the logarithmic growth phase at a density of $5 \times 10^{4} /$ well and cultured overnight. After rewarming, $10 \mu \mathrm{l}$ CCK-8 (Abcam) was added to each cell group and cells were incubated for $1 \mathrm{~h}$. The absorbance of cells at $450 \mathrm{~nm}$ was measured by a microplate micrograph.

Colony formation assay. Cells in the logarithmic growth phase of each group were digested with EDTA $+0.25 \%$ trypsin and blown into single cells, and suspended in DMEM culture solution of $10 \%$ fetal bovine serum (FBS; Gibco; Thermo Fisher Scientific, Inc.). A total of 200 cells were inoculated into a $10 \mathrm{~cm}$ cell culture dish containing $10 \mathrm{ml} \mathrm{DMEM} \mathrm{+} \mathrm{10 \%} \mathrm{FBS,}$ and the cells were evenly dispersed by gentle rotation. The cells were placed in an incubator at $37^{\circ} \mathrm{C}, 5 \% \mathrm{CO}_{2}$ and saturated humidity for 10-14 days. The cell growth was observed in time during the culture, and the culture was stopped when the macroscopic clones appeared in the culture dish. The supernatant was discarded and carefully washed twice with PBS. The cells were fixed with $5 \mathrm{ml}$ of methanol for $15 \mathrm{~min}$ at room temperature and then stained using Giemsa solution for 15-30 min at room temperature. Each treatment run triplicate, and the colonies were counted with an optical microscope (Olympus Corporation) at x10 magnification.

Cell cycle detection using flow cytometry. PASMCs cultured in each group were digested with $0.25 \%$ trypsin (without EDTA), and the cells were digested to obtain a single-cell suspension. The cells were collected by centrifugation (at $200 \mathrm{x} \mathrm{g}$ ) for 5 min at $4^{\circ} \mathrm{C}$, and washed twice in pre-chilled PBS, and then fixed overnight at $4^{\circ} \mathrm{C}$ with $70 \%$ ethanol. After the fixation, the cells were treated with $500 \mu \mathrm{l}$ PBS containing $50 \mu \mathrm{g} / \mathrm{ml}$ propidium iodide (PI), $100 \mu \mathrm{g} / \mathrm{ml}$ RNase A, $0.2 \%$ Triton X-100, and incubated for $30 \mathrm{~min}$ in the dark at $4^{\circ} \mathrm{C}$. The processed samples were detected by flow cytometry (CytoFLEX LX; Beckman 
Coulter, Inc.). The data analysis was calculated using FlowJo software (FlowJo LLC).

Western blotting. The PASMCs in each group were cultured overnight and then lysed using RIPA buffer. The total protein was extracted in RIPA buffer, separated on polyacrylamide gels (30 $\mu \mathrm{g}$ per well), and immobilized on polyvinylidene fluoride (PVDF) membrane. After blocking with 5\% non-fat milk at room temperature for $1.5 \mathrm{~h}$, the membranes were incubated at $4^{\circ} \mathrm{C}$ overnight with primary antibodies; when PVDF membranes were used, they were washed three times with PBST $(0.5 \%$ Tween-20 in PBS). Following washing in PBST, the membranes were treated with horseradish peroxide (HRP)-conjugated goat anti-rabbit IgG; (1:5,000; cat. no. ab205718) or goat anti-mouse IgG H\&L (1:5,000; cat. no. ab205719; Abcam) secondary antibodies at room temperature for $2 \mathrm{~h}$. Finally, an enhanced chemiluminescence kit (Beijing Solarbio Science \& Technology Co., Ltd.) was used to treat the membrane for visualization. The following primary antibodies were used in the study (all from Abcam): Anti-cyclin D1 (1:2,000; cat. no. ab134175), anti-CDK4 (1:1,000; cat. no. ab108357), anti-PCNA (1:1,000; cat. no. ab29), anti-NFATc2 $(1: 1,000$; cat. no. ab169140), anti-NFATc4 (1:1,000; cat. no. ab3447), anti- $\beta$-actin (1:1,000; cat. no. ab8227) and anti-laminin B1 (1:5,000; cat. no. ab256380).

The isolation of nuclear and cytoplasmic fractions was carried out according to the REAP method (23). Briefly, cells were washed twice with ice-cold PBS, and the cell pellet was lysed in $0.1 \%$ NP-40 PBS lysis buffer. The nuclei were then isolated by differential centrifugation at $10,000 \mathrm{x}$ for $10 \mathrm{sec}$ at $4^{\circ} \mathrm{C}$, and the supernatant was retained as the cytoplasmic fraction. For western blotting, the nuclei were sonicated in $0.1 \%$ NP-40 PBS lysis buffer for further analysis. The samples were kept on ice during sonication, and the frequency was $20 \mathrm{kHz}, 2$ pulses, $8 \mathrm{sec}$.

Wound healing assay. Horizontal lines were drawn across the well with a marker pen and ruler on the back of the 6-well plate. Cell suspensions at a concentration of $1 \times 10^{6} / \mathrm{ml}$ were prepared, and $1 \times 10^{5} / \mathrm{ml}$ cells were added to each well. Vertical lines were scratched with a smaller pipette tip and ruler. Then, the cells were washed three times in PBS after which the floating cells were removed and serum-free medium was added. These cells were cultured at $37^{\circ} \mathrm{C}$ with $5 \% \mathrm{CO}_{2}$ for $24 \mathrm{~h}$ and images were obtained thereafter (24), using an optical microscope (Olympus Corporation) at $\mathrm{x} 40$ magnification. The quantification was calculated with ImageJ software (v 1.80, National Institutes of Health).

Reverse transcription-quantitative $R T-q P C R$. Total RNA was extracted using TRIzol ${ }^{\circledR}$ reagent (Invitrogen; Thermo Fisher Scientific, Inc.). The purity and concertation of extracted RNA were determined by the NanoDrop ${ }^{\mathrm{TM}}$ ND-1000 (Thermo Fisher Scientific, Inc.). PrimeScript ${ }^{\mathrm{TM}}$ RT reagent Kit (Takara Bio, Inc.) was used to prepare cDNA according to the manufacturer's instructions. RT-qPCR was carried out using the SYBR Green PCR Master Mix (Takara Bio, Inc.), under the following conditions: $94^{\circ} \mathrm{C}$ for $5 \mathrm{~min}$, followed by 32 cycles of $94^{\circ} \mathrm{C}$ for $40 \mathrm{sec}, 57^{\circ} \mathrm{C}$ for $40 \mathrm{sec}$ and $72^{\circ} \mathrm{C}$ for $1 \mathrm{~min}$, with a final elongation step at $72^{\circ} \mathrm{C}$ for $5 \mathrm{~min}$. RT-qPCR was performed in
Table I.List of primers used in reverse transcription-quantitative PCR.

\begin{tabular}{ll} 
Gene name & \multicolumn{1}{c}{ Primer sequences $\left(5^{\prime} \rightarrow 3^{\prime}\right)$} \\
\hline NFATc1 & F: CACTCAGAAAATCCCAACTTACC \\
& R: CAGGTTTGGGGTCATTCTGC \\
NFATc2 & F: CAGGCAGGTTCACAGGTGTG \\
& R: CTGAGATGTCCTGCAACAACC \\
NFATc3 & F: CTGCTGCTGCTGGTGAGATC \\
& R: GGTAAAGAAGGCCCCTACCATC \\
NFATc4 & F: GGCTACAATGAGAAGCCATTG \\
& R: CTTCAGGATTCCAGCACAGTC \\
NFAT5 & F: CAAACCAGAACCCGATGGC \\
& R: GTGACCCTTGAACCAACTGG
\end{tabular}

F, forward; R, reverse; NFAT, nuclear factor of activated T cells.

triplicate, and the expression of RNA was calculated using the $2^{-\Delta \Delta \mathrm{Cq}}$ method (25). mRNA expression was normalized against GAPDH, allowing comparison of mRNA levels. The primers used in this study are listed in Table I.

Transwell migration assay. Cell migration and invasion were measured using Transwell migration assay as previously described (26). Briefly, $400 \mathrm{ml}$ culture medium (DMEM + 1\% FBS; Gibco; Thermo Fisher Scientific, Inc.) and $600 \mathrm{ml}$ complete medium (DMEM + 10\% FBS; Gibco; Thermo Fisher Scientific, Inc.) were separately added to the upper and lower chambers, respectively; $5 \times 10^{4}$ cells $/ \mathrm{ml}$ were plated in the upper well. After incubation for $36 \mathrm{~h}$ at $37^{\circ} \mathrm{C}$, a cotton-tipped swab was used to remove the non-invading cells away from the upper surface of the membrane. Following which, the invading cells were fixed with methanol for $10 \mathrm{~min}$ and stained with $0.1 \%$ crystal violet hydrate for $5 \mathrm{~min}$ at room temperature. The stained cells were counted as cells per field using an optical microscope (Olympus Corporation) at x10 magnification, at least three fields were observed for each experiment.

Small interfering (si)RNA. PASMCs were transfected with non-targeting control siRNA or NFATc2 siRNA using Lipofectamine ${ }^{\circledR} 2000$ reagent (Thermo Fisher Scientific, Inc.). The following primer sequences were used: NFATc2 siRNA sense, 5'-UCAGUAAACACAACUUUGGACCC-3' and antisense, 5'-CCAAAGUUGUGUUUACUGAGAUU-3'; and negative control siRNA sense, 5'-UUCUCCGAACGUGUC ACGUTT-3' and antisense, 5'-ACGUGACACGUUCGGAGA ATT-3'. Non-targeting control siRNA and NFATc2 siRNA sequences were purchased from Ambion (Thermo Fisher Scientific, Inc.). The concentration of control siRNA and NFATc2 siRNA was 60-80 nM. Following transfection for $\sim 24 \mathrm{~h}$, the PASMCs were used in RT-qPCR, Transwell, colony formation and western blotting assays.

Statistical analysis. SPSS version 26.0 (IBM Corp.) and GraphPad Prism 8 (GraphPad Software, Inc.) statistical software packages were used for data analysis. ImageJ 

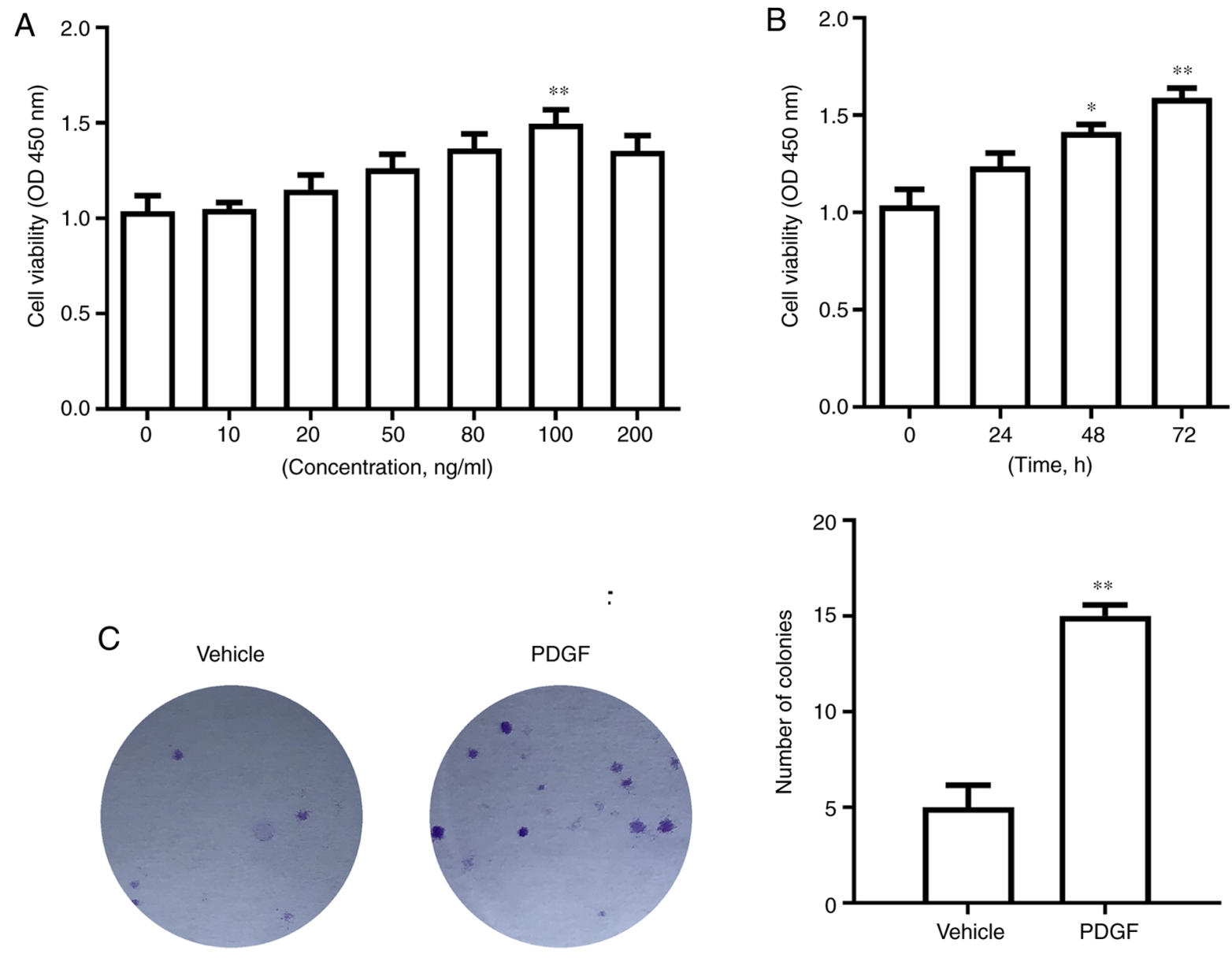

Figure 1. PASMC viability and colony formation after PDGF stimulation. (A) The dose-dependent effect of PDGF on PASMC viability $48 \mathrm{~h}$ after treatment. ${ }^{* *} \mathrm{P}<0.01$ vs. $0 \mathrm{ng} / \mathrm{ml}$. (B) The time-dependent effect of PDGF on PASMC viability after treatment with $100 \mathrm{ng} / \mathrm{ml} \mathrm{PDGF}$. ${ }^{*} \mathrm{P}<0.05$ and ${ }^{* *} \mathrm{P}<0.01 \mathrm{vs}$. 0 h. (C) Colony formation assay. ${ }^{* *} \mathrm{P}<0.01$ vs. Vehicle group. Each experiment was conducted in triplicate. PASMC, pulmonary arterial smooth muscle cell; PDGF, platelet-derived growth factor.

(version 1.80; National Institutes of Health) was used to performed semi-quantitative analysis. All experiments were conducted at least three times. The results are presented as the mean \pm standard deviation. Student's t-test or one-way analysis of variance followed by a Tukey's post hoc test were used to calculate the statistical differences. $\mathrm{P}<0.05$ was considered to indicate a statistically significant difference.

\section{Results}

PDGF enhances PASMC viability and proliferation. PASMCs were treated with different concentrations of PDGF for $72 \mathrm{~h}$. The CCK-8 experiments showed that as PDGF concentration increased, PASMC viability increased. When the concentration of PDGF reached $100 \mathrm{ng} / \mathrm{ml}$, PASMC viability reached its peak compared with $0 \mathrm{ng} / \mathrm{ml}$ $(\mathrm{P}<0.01$; Fig. 1A). At the same time, following treatment with $100 \mathrm{ng} / \mathrm{ml}$ PDGF for $72 \mathrm{~h}$, PASMC viability was significantly higher compared with that of the $0 \mathrm{~h}$ treatment group $(\mathrm{P}<0.01$; Fig. 1B). After treatment with $100 \mathrm{ng} / \mathrm{ml}$ PDGF, cell proliferation increased, and the number of colonies increased significantly compared with the vehicle group $(\mathrm{P}<0.01$; Fig. 1C).
PDGF promotes division of PASMCs. Compared with the blank and vehicle groups, the PDGF-treated group showed a significantly increased proportion of PASMCs in the synthesis $(\mathrm{S})$ phase $(\mathrm{P}<0.01$; Fig. $2 \mathrm{~A})$. Western blotting was used to detec $\mathrm{T}$ cell cycle-related proteins. The relative expression levels of cyclin D1, CDK4 and PCNA in the PDGF-treated group were significantly higher than in the vehicle group $(\mathrm{P}<0.01$; Fig. 2B).

PDGF enhances PASMC migration. Wound healing and Transwell migration assays were used to detect cell migration following treatment with PDGF. After $24 \mathrm{~h}$ of PDGF treatment, the wound closure rate was significantly higher than in the vehicle group (Fig. 3A), and the results of the Transwell migration assay showed that the number of migrated cells in the PDGF group was significantly higher than that in the vehicle group (Fig. 3B). The aforementioned results indicated that PDGF treatment increased PASMC migration.

Effects of PDGF on the expression of NFATc1-5 in PASMCs. The NFAT transcription factor family consists of five members, namely NFATc1, NFATc2, NFATc3, NFATc4 and NFATc5 (27). The mRNA expression levels of all five members 
A
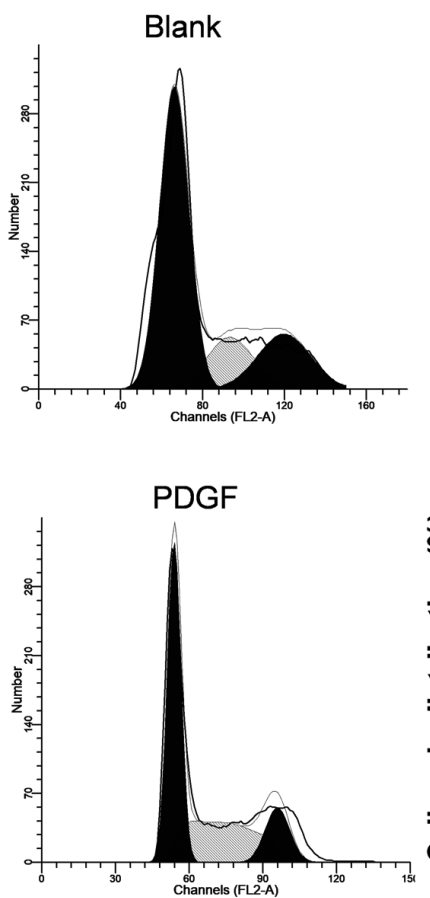
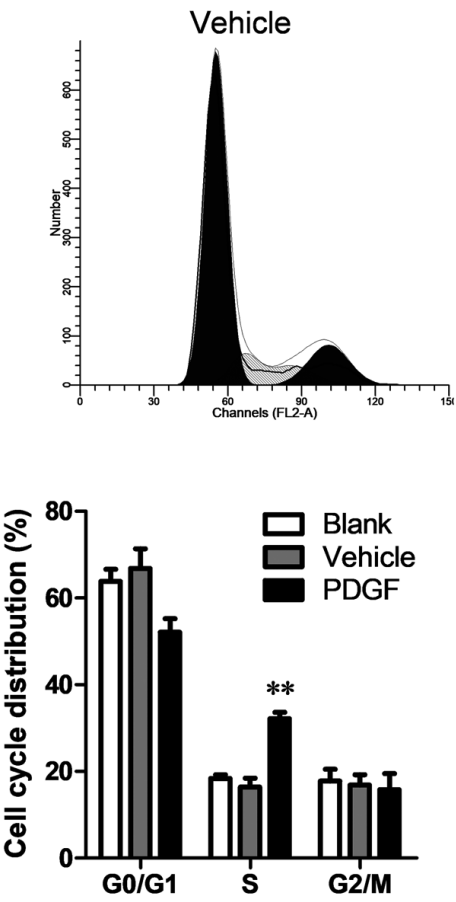

B
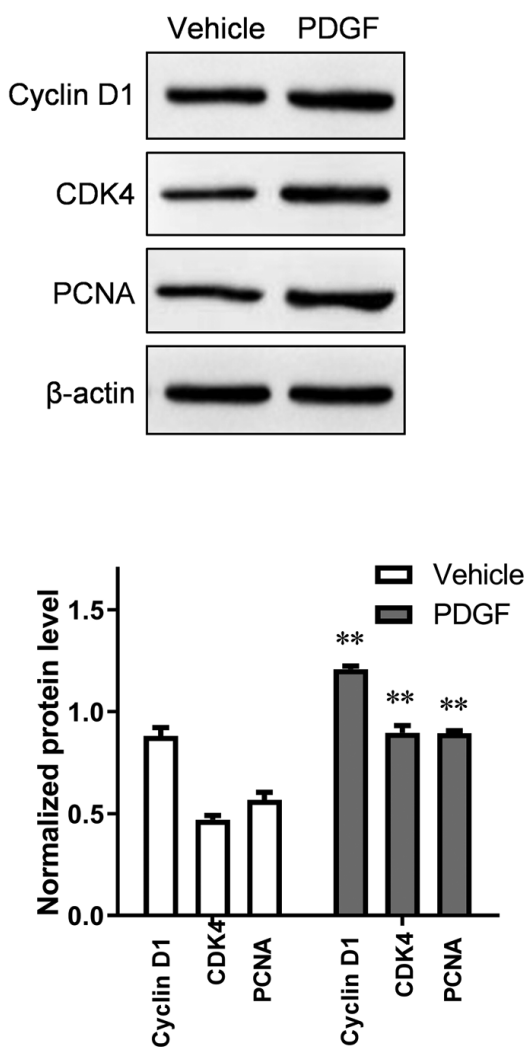

Figure 2. Cell cycle distribution of pulmonary arterial smooth muscle cells following treatment with PDGF. (A) PDGF increases the number of cells in the $\mathrm{S}$ phase of the cell cycle. (B) The expression levels of cell cycle-related proteins, as assessed by western blotting. The gray-scale value of the protein is shown at the bottom. ${ }^{* *} \mathrm{P}<0.01$ vs. Corresponding blank and vehicle groups. PDGF, platelet-derived growth factor; PCNA, proliferating cell nuclear antigen.

A
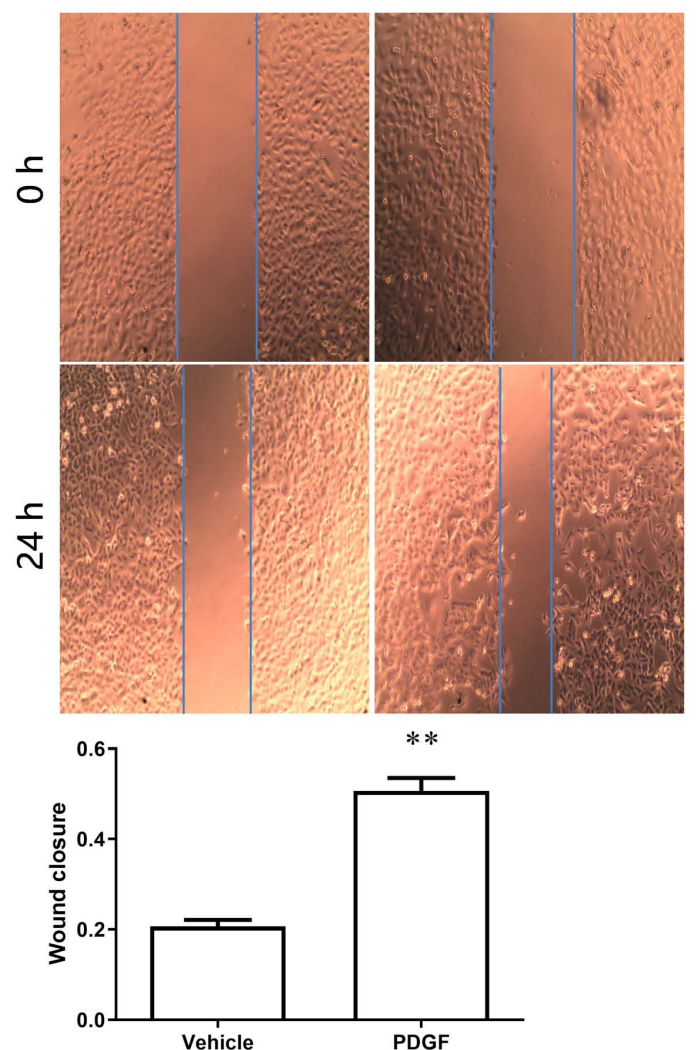

B
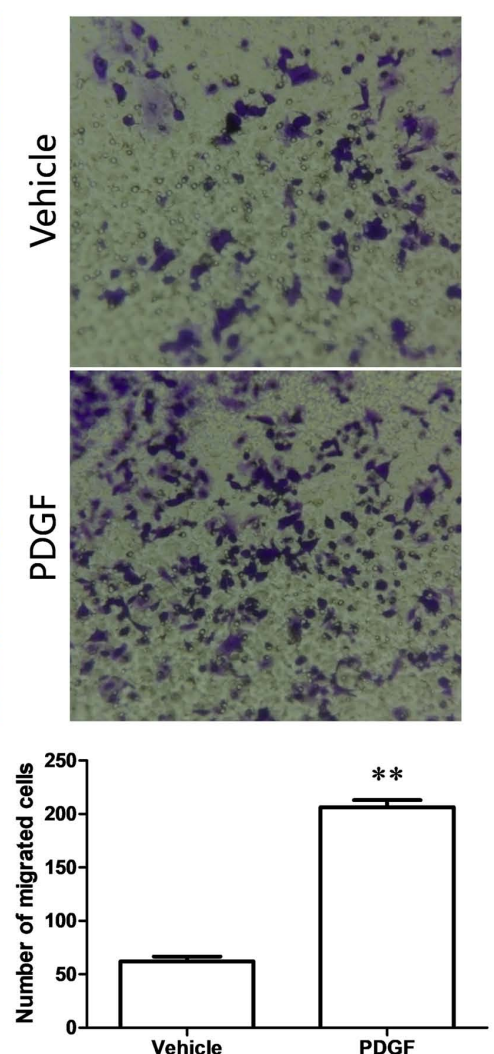

Figure 3. Cell migration of pulmonary arterial smooth muscle cells following treatment with PDGF. (A) Wound healing and (B) Transwell migration assays. The bars represent the mean $\pm \mathrm{SD}$ of three independent experiments. ${ }^{* *} \mathrm{P}<0.01$ vs. Vehicle group. PDGF, platelet-derived growth factor. 

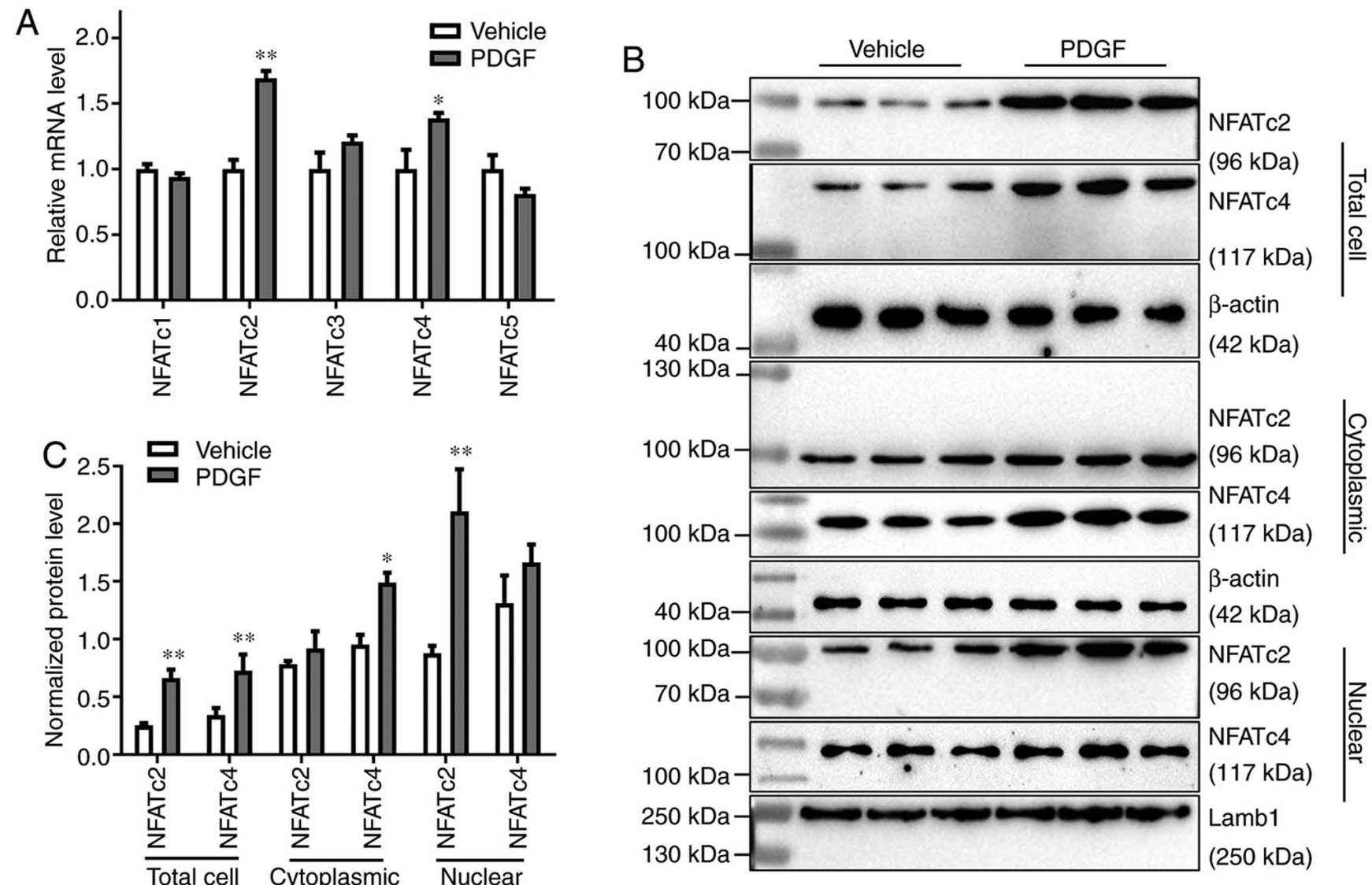

Figure 4. PDGF increases the expression of NFATc2/4 in PASMCs. (A) Reverse transcription-quantitative PCR showed the alterations in the expression levels of NFAT mRNA in normal and PDGF-treated PASMCs. NFATc2, NFATc3 and NFATc4 mRNA expression was increased in PDGF-treated cells. (B and C) Western blot analysis of NFATc2/NFATc4 from total cell lysates, cytoplasm and nuclear fractions in normal and PDGF-treated PASMCs. $\beta$-actin and lamb1 proteins were used as the loading controls. ${ }^{*} \mathrm{P}<0.05$ and ${ }^{* *} \mathrm{P}<0.01$ vs. Vehicle group. PDGF, platelet-derived growth factor; lamb1, laminin $\mathrm{B} 1$; PASMC, pulmonary arterial smooth muscle cell; NFAT, nuclear factor of activated T cells.

in PDGF-treated PASMCs were detected by RT-qPCR. The relative mRNA expression levels of NFATc2 and NFATc4 were significantly increased after PDGF treatment $(\mathrm{P}<0.05$; Fig. 4A). To compare the protein expression levels of NFATc2 and NFATc4 in different parts of the cell, western blotting was used to detect the expression of NFATc2 and NFATc4 in the total cell, cytoplasm and nucleus following PDGF treatment. Compared with the vehicle group, the expression of NFATc2 in the nucleus and total cell increased significantly $(\mathrm{P}<0.01)$; although an increase was also detected in cytoplasmic levels, this was not significant. NFATc4 expression was significantly increased in total cell and cytoplasm $(\mathrm{P}<0.05)$, but the expression did not show a significant change in the nucleus (Fig. 4B and C).

Roles of NFATc2 on PDGF-induced proliferation and migration of PASMCs. To evaluate the role of NFATc2 in PDGF-induced cell proliferation and migration, cells pretreated with NFAT pathway inhibitors, CsA or siNFATc2, were treated with PDGF, and then western blotting, RT-qPCR, colony formation and Transwell assays were performed to evaluate changes in cell proliferation and migration. As shown in Fig. 5A, the number of colonies following $30 \mu \mathrm{M}$ CsA treatment was significantly lower than after $0 \mu \mathrm{M} \mathrm{CsA}$ treatment $(\mathrm{P}<0.01)$. Furthermore, the number of colonies in the PDGF-treated group also significantly decreased after the addition of $30 \mu \mathrm{M}$ CsA $(\mathrm{P}<0.01)$. The results of the cell migration experiments were similar to those of the colony formation assay. Following $30 \mu \mathrm{M}$ CsA treatment, cell migration in the PDGF and vehicle group decreased significantly ( $\mathrm{P}<0.01$; Fig. 5B).

After the cells were pretreated with the NFAT pathway inhibitor siNFATc2, RT-qPCR was used to detect the relative expression of NFATc 2 mRNA. The relative expression of mRNA in the siNFATc2 group decreased significantly $(\mathrm{P}<0.01$; Fig. 6A). Additionally, western blotting demonstrated that following transfection with siNFATc2, the expression of NFATc2 significantly decreased in PASMCs (Fig. 6B). The normalized protein level of PCNA in cells pretreated with siNFATc2 in the vehicle and PDGF groups was significantly lower than that in the siNC group $(\mathrm{P}<0.01$; Fig. 6C). Colony formation experiments were performed to further examine the effect of siNFATc2 on the proliferation of PASMCs. In both the vehicle and PDGF groups, the number of colonies following siNFATc2 treatment was significantly reduced $(\mathrm{P}<0.01$; Fig. $6 \mathrm{D})$. A Transwell assay was used to detecT cell migration. Transfection with siNFATc2 significantly reduced the number of migrated cells in the PDGF group $(\mathrm{P}<0.01)$, but had no significant effect on the vehicle group (Fig. 6E). 
A
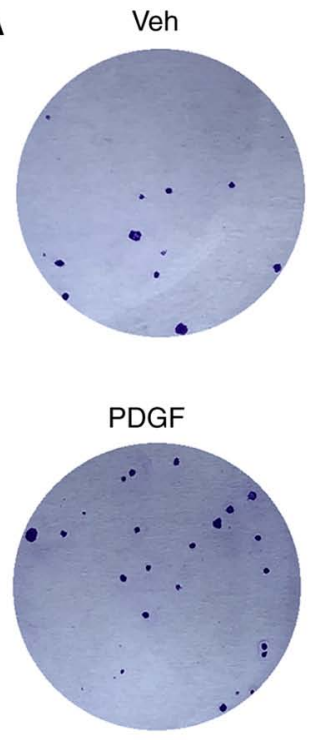

B
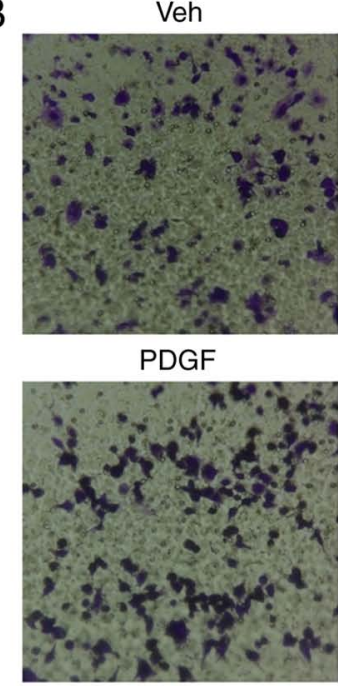
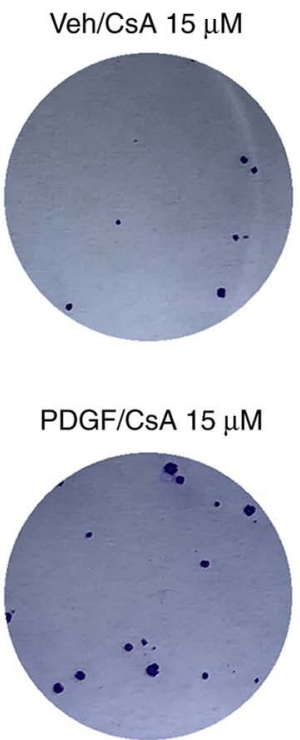

Veh/CsA $15 \mu \mathrm{M}$

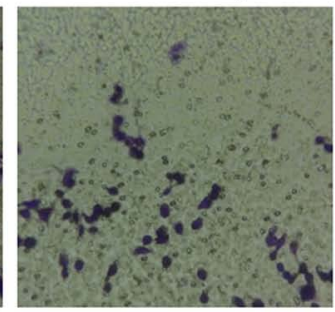

PDGF/CsA $15 \mu \mathrm{M}$

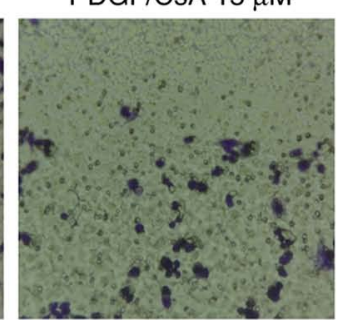

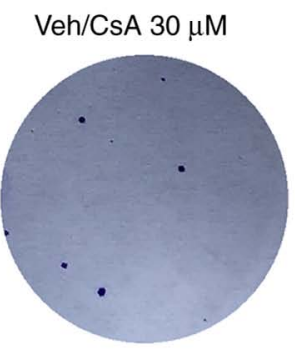

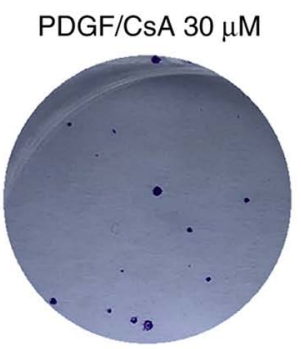

Veh/CsA $30 \mu \mathrm{M}$

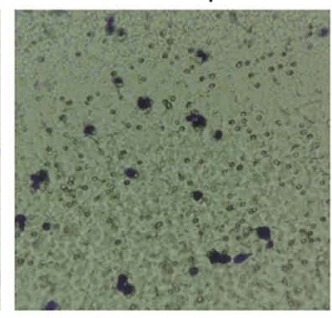

PDGF/CsA $30 \mu \mathrm{M}$

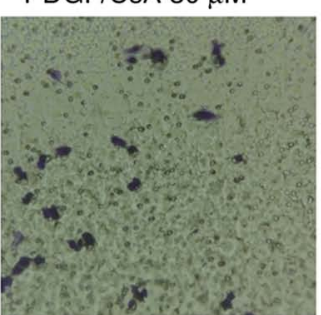

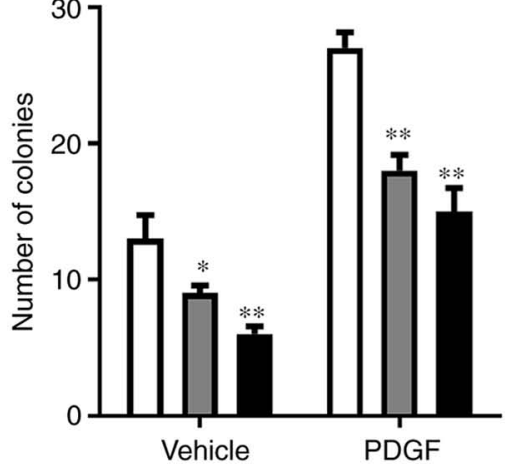

Figure 5. Proliferation and migration of PASMCs following pretreatment with different concentrations of CsA in vehicle and PDGF groups. (A) The colony formation assay showed the effect of CsA on the proliferation of PASMCs. (B) The Transwell assay showed the number of migrated cells in each group. ${ }^{*} \mathrm{P}<0.05$ and ${ }^{* *} \mathrm{P}<0.01$ vs. Vehicle group. PDGF, platelet-derived growth factor; PASMC, pulmonary arterial smooth muscle cell; CsA, cyclosporin A.

\section{Discussion}

PAH is a serious condition characterized by increased pulmonary circulatory resistance and pulmonary artery remodeling, which can eventually lead to right ventricular failure and death (28). The increased pulmonary vascular resistance in PAH is partly due to increased proliferation of PASMCs (29). PDGF, as an important mitogenic promoter, has been demonstrated to promote PASMC proliferation and migration (30), which is consistent with the present results. The results of the current study showed thaT cell viability was highest when cells were treated with $100 \mathrm{ng} / \mathrm{ml}$ PDGF. PDGF-treated PASMCs were analyzed using flow cytometry. The number of cells in the $S$ phase was significantly higher than that in the vehicle and blank groups. To verify this result, western blotting was performed to determine the expression of cell cycle-related proteins. The results verified that PDGF increased PASMC division. Furthermore, to investigate the effect of PDGF on PASMC migration, wound healing and Transwell migration assays were conducted after PDGF treatment. Migration of PASMCs after PDGF treatment was significantly increased. Several growth factors, such as PDGF, fibroblast growth factor 2 and epidermal growth factor, are implicated in abnormal PASMC proliferation and migration, which contributes to pulmonary vascular remodeling in PAH (31). This finding was consistent with the present results of PDGF.

It is commonly known that intrinsic changes in $\mathrm{Ca}^{2+}$ homeostasis in PASMCs plays an important role in pulmonary vasoconstriction and vascular reconstruction in PAH (32) Of note, it was previously discovered that store-operated $\mathrm{Ca}^{2+}$ entry (SOCE) is the main channel that regulates $\mathrm{Ca}^{2+}$ influx in PASMCs and regulates PASMC proliferation, apoptosis and migration in PAH $(33,34)$. The CaN/NFAT pathway is 

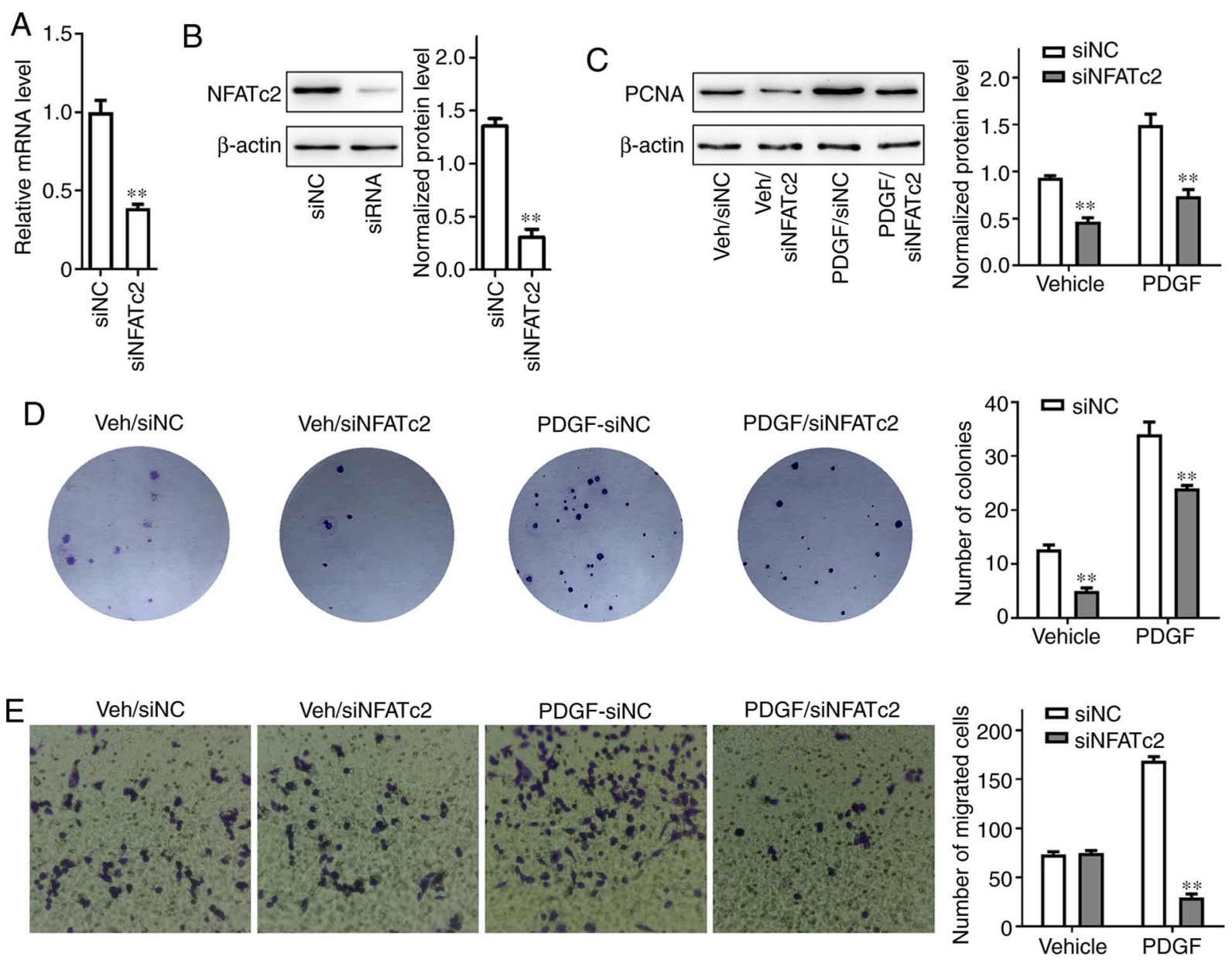

Figure 6. Proliferation and migration of PASMCs following pretreatment with different concentrations of siNFATc2 in vehicle and PDGF groups. Analysis of (A) mRNA and (B) protein expression levels following transfection of PASMCs with siNFATc2. (C) Protein expression levels of PCNA in cells pretreated with siNFATc2 in the vehicle and PDGF groups. (D) The colony formation assay showed the effect of siNFATc2 on the proliferation of PASMCs. (E) The Transwell migration assay of PASMCs transfected with siNFATc2 in vehicle or PDGF-treated PASMCs. The bars represent the mean \pm SD of three independent experiments. ${ }^{* *} \mathrm{P}<0.01$ vs. siNC group. PDGF, platelet-derived growth factor; PASMC, pulmonary arterial smooth muscle cell; PCNA, proliferating cell nuclear antigen; siNFATc2, small RNA interfering of nuclear factor of activated T cells, Cytoplasmic 2; NC, negative control.

the most important downstream signaling pathway of SOCE and is involved in numerous physiological and pathological processes (32). An increase in cytosolic free $\mathrm{Ca}^{2+}$ concentration $\left[\left(\mathrm{Ca}^{2+}\right) \mathrm{cyt}\right]$ in PASMCs is a major trigger for pulmonary vasoconstriction and an important underlying mechanism of pulmonary vascular remodeling via stimulation of PASMC proliferation and inhibition of PASMC apoptosis (35).

Based on the aforementioned reports, in the present study it was speculated that PDGF may regulate NFAT by increasing the $\mathrm{Ca}^{2+}$ concentration, which would eventually lead to the occurrence of PAH. RT-qPCR was performed to detect the mRNA expression levels of NFATc1, NFATc2, NFATc3, NFATc4 and NFAT5 in PASMCs treated with PDGF. Following PDGF treatment, the relative expression levels of NFATc2 and NFATc4 mRNA were significantly higher than those in the vehicle group. As a nuclear factor, NFAT can only play a role once it is transferred into the nucleus (36). When the relative expression of NFATc2 and NFATc 4 proteins were compared in different parts of the cell it was revealed that the expression of NFATc2 in total cell lysates and the nucleus was significantly increased. Although NFATc4 was notably increased in the nucleus, there was no significant change in the relative expression; hence, NFATc2 was chosen as the research target and investigated in the following assays.

Colony formation and cell migration assays were performed to verify the role of NFATc2 in PDGF-induced PASMC proliferation and migration. Additionally, $\mathrm{Ca}^{2+}$-NFAT signaling inhibitor CsA was used to treat PASMCs in each group. The results showed that the number of colonies was significantly reduced following the addition of $30 \mu \mathrm{M} \mathrm{CsA}$, compared with the vehicle group, indicating that CsA could reduce the proliferation of PASMCs. To verify whether CsA treatment significantly reduced PASMC migration, PASMCs were transfected with siNFATc2. The results showed that, compared with siNC, the proliferative and migratory abilities of PASMCs were significantly reduced following transfection with siNFATc2. However, the findings of the colony formation assays showed inconsistent effects of NFATc2 inhibition, as PDGF is supposed to reduce number of colonies in the presence of CsA or siNFATc2. This difference was primarily due to the fact that PGDF can be influenced by other signaling pathways, and PGDF can regulate cell adherence via the signaling 
pathway independent of NFATc2, such as the Akt/mTOR signaling pathway (37). According to a previous study by Ogawa et al (38), PDGF upregulates stromal interaction molecule 1 (STIM1)/calcium release-activated calcium channel protein 1 (Orai1) via the Akt/mTOR signaling pathway in PASMCs, thereby enhancing $\mathrm{Ca}^{2+}$ entry.

At present, drugs that target endothelin are the first choice in the treatment of PAH (39). During development, endothelial cells secrete the polypeptide platelet-derived growth factor (PDGF). As endothelial cells differentiate and undergo tubulogenesis, secreted PDGF is thought to form a concentration gradient, which is sensed by surrounding smooth muscle precursors via the tyrosine kinase receptor PDGFR- $\beta$ to promote their migration and proliferation, resulting in recruitment and assembly of the vessel wall.

The present study showed that in PDGF-treated rat PASMCs, the relative expression of NFATc2 in the nucleus changed significantly compared with vehicle cells, and the proliferative and migratory abilities of rat PASMCs was significantly reduced following treatment with NFATc2 inhibitors. In conclusion, this study indicated that PDGF mediated PASMC proliferation and migration by regulating NFATc2, which could be used as a potential target for PAH treatment.

\section{Acknowledgements}

Not applicable.

\section{Funding}

The present research was funded by grants from the 'Special and Joint Program' of Yunnan Provincial Science and Technology Department and Kunming Medical University [grant nos. 2018FE001(-082), 2018FE001(-206)], the Yunnan Health Training Project of High Level Talents (grant nos. H-2019026 and H-2018095), and the Young Academic and Technical Leaders of Yunnan Province (grant no. 2017HB053).

\section{Availability of data and materials}

All data generated or analyzed during this study are included in this manuscript.

\section{Authors' contributions}

FYZ and SLX performed the experiments, interpreted the data, and were major contributors in writing the manuscript. CFZ, JL and YZ were responsible for data analysis and visualization. JY and XQX significantly contributed to the design and conception of the study. All authors read and approved the final manuscript.

\section{Ethics approval and consent to participate}

The present study was approved by the Ethics Committee of The Fourth Affiliated Hospital of Kunming Medical University, The Second People's Hospital of Yunnan Province (Kunming, China).

\section{Patient consent for publication}

Not applicable.

\section{Competing interests}

The authors declare that they have no competing interests.

\section{References}

1. Rubin LJ, Badesch DB, Barst RJ, Galie N, Black CM, Keogh A, Pulido T, Frost A, Roux S, Leconte I, et al: Bosentan therapy for pulmonary arterial hypertension. N Engl J Med 346: 896-903, 2002.

2. Humbert M, Deng Z, Simonneau G, Barst RJ, Sitbon O, Wolf M, Cuervo N, Moore KJ, Hodge SE, Knowles JA and Morse JH: BMPR2 germline mutations in pulmonary hypertension associated with fenfluramine derivatives. Eur Respir J 20: 518-523, 2002.

3. Humbert M, Sitbon O and Simonneau G: Treatment of pulmonary arterial hypertension. N Engl J Med 351: 1425-1436, 2004.

4. Walker J, Undem C, Yun X, Lade J, Jiang H and Shimoda LA: Role of Rho kinase and $\mathrm{Na}^{+} / \mathrm{H}^{+}$exchange in hypoxia-induced pulmonary arterial smooth muscle cell proliferation and migration. Physiol Rep 4: e12702, 2016.

5. Hou X, Chen J, Luo Y, Liu F, Xu G and Gao Y: Silencing of STIM1 attenuates hypoxia-induced PASMCs proliferation via inhibition of the SOC/Ca ${ }^{2+} / \mathrm{NFAT}$ pathway. Respir Res 14: 2, 2013.

6. Heldin $\mathrm{CH}$ and Westermark B: Mechanism of action and in vivo role of platelet-derived growth factor. Physiol Rev 79: 1283-1316, 1999.

7. Cimminiello C, Arpaia G, Aloisio M, Uberti T, Rossi F, Pozzi F and Bonfardeci G: Platelet-derived growth factor (PDGF) in patients with different degrees of chronic arterial obstructive disease. Angiology 45: 289-293, 1994.

8. Perros F, Montani D, Dorfmüller P, Durand-Gasselin I, Tcherakian C, Le Pavec J, Mazmanian M, Fadel E, Mussot S, Mercier O, et al: Platelet-derived growth factor expression and function in idiopathic pulmonary arterial hypertension. Am J Respir Crit Care Med 178: 81-88, 2008.

9. Antoniu SA: Targeting PDGF pathway in pulmonary arterial hypertension. Expert Opin Ther Targets 16: 1055-1063, 2012.

10. Schermuly RT, Dony E, Ghofrani HA, Pullamsetti S, Savai R, Roth M, Sydykov A, Lai YJ, Weissmann N, Seeger W and Grimminger F: Reversal of experimental pulmonary hypertension by PDGF inhibition. J Clin Invest 115: 2811-2821, 2005.

11. Macian F: NFAT proteins: Key regulators of T-cell development and function. Nat Rev Immunol 5: 472-484, 2005.

12. Dorfmüller P, Perros F, Balabanian K and Humbert M: Inflammation in pulmonary arterial hypertension. Eur Respir J 22: 358-363, 2003.

13. Voelkel NF, Cool C, Lee SD, Wright L, Geraci MW and Tuder RM: Primary pulmonary hypertension between inflammation and cancer. Chest 114 (Suppl 3): 225S-230S, 1998.

14. Vihma H, Pruunsild $P$ and Timmusk T: Alternative splicing and expression of human and mouse NFAT genes. Genomics 92: 279-291, 2008.

15. Senavirathna LK, Huang C, Yang X, Munteanu MC, Sathiaseelan R, Xu D, Henke CA and Liu L: Hypoxia induces pulmonary fibroblast proliferation through NFAT signaling. Sci Rep 8: 2709, 2018.

16. Bonnet S, Rochefort G, Sutendra G, Archer SL, Haromy A, Webster L, Hashimoto K, Bonnet SN and Michelakis ED: The nuclear factor of activated $\mathrm{T}$ cells in pulmonary arterial hypertension can be therapeutically targeted. Proc Natl Acad Sci USA 104: 11418-11423, 2007.

17. Chen R, Yan J, Liu P, Wang Z, Wang C, Zhong W and Xu L: The role of nuclear factor of activated $\mathrm{T}$ cells in pulmonary arterial hypertension. Cell Cycle 16: 508-514, 2017.

18. MacLean MMR: The serotonin hypothesis in pulmonary hypertension revisited: Targets for novel therapies (2017 Grover conference series). Pulm Circ 8: 2045894018759125, 2018.

19. Maroteaux L, Ayme-Dietrich E, Aubertin-Kirch G, Banas S, Quentin E, Lawson R and Monassier L: New therapeutic opportunities for 5- $\mathrm{HT}_{2}$ receptor ligands. Pharmacol Ther 170: 14-36, 2017. 
20. Han J, Tian H, Liu Y and Fan F: Sarpogrelate attenuates pulmonary arterial hypertension via calcium/calcineurin axis. Front Biosci (Landmark Ed) 24: 607-615, 2019.

21. Hassoun PM, Mouthon L, Barberà JA, Eddahibi S, Flores SC, Grimminger F, Jones PL, Maitland ML, Michelakis ED, Morrell NW, et al: Inflammation, growth factors, and pulmonary vascular remodeling. J Am Coll Cardiol 54 (Suppl 1): S10-S19, 2009.

22. Yao C, Yu J, Taylor L, Polgar P, McComb ME and Costello CE: Protein expression by human pulmonary artery smooth muscle cells containing a BMPR2 mutation and the action of ET-1 as determined by proteomic mass spectrometry. Int J Mass Spectrom 378: 347-359, 2015.

23. Nabbi A and Riabowol K: Rapid isolation of nuclei from cells in vitro. Cold Spring Harb Protoc 2015: 769-772, 2015.

24. Rodriguez LG, Wu X and Guan JL: Wound-healing assay. Methods Mol Biol 294: 23-29, 2005.

25. Livak KJ and Schmittgen TD: Analysis of relative gene expression data using real-time quantitative PCR and the 2(-Delta Delta C(T)) method. Methods 25: 402-408, 2001.

26. Gong L, Zhu L, Wang S and Zhang Z: Transthyretin regulates the migration and invasion of JEG-3 cells. Oncol Lett 13: 1242-1246, 2016.

27. Vihma H, Luhakooder M, Pruunsild $\mathrm{P}$ and Timmusk $\mathrm{T}$ : Regulation of different human NFAT isoforms by neuronal activity. J Neurochem 137: 394-408, 2016.

28. Fukumoto Y, Tawara S and Shimokawa H: Recent progress in the treatment of pulmonary arterial hypertension: Expectation for rho-kinase inhibitors. Tohoku J Exp Med 211: 309-320, 2007.

29. Nie X, Chen Y, Tan J, Dai Y, Mao W, Qin G, Ye S, Sun J, Yang Z and Chen J: MicroRNA-221-3p promotes pulmonary artery smooth muscle cells proliferation by targeting AXIN2 during pulmonary arterial hypertension. Vascul Pharmacol 116: 24-35, 2019.

30. Sysol JR, Natarajan V and Machado RF: PDGF induces SphK1 expression via Egr-1 to promote pulmonary artery smooth muscle cell proliferation. Am J Physiol Cell Physiol 310: C983-C992, 2016.

31. Pullamsetti SS, Savai R, Seeger W and Goncharova EA Translational advances in the field of pulmonary hypertension. From cancer biology to new pulmonary arterial hypertension therapeutics. Targeting cell growth and proliferation signaling hubs. Am J Respir Crit Care Med 195: 425-437, 2017.
32. He RL, Wu ZJ, Liu XR, Gui LX, Wang RX and Lin MJ: Calcineurin/NFAT signaling modulates pulmonary artery smooth muscle cell proliferation, migration and apoptosis in monocrotaline-induced pulmonary arterial hypertension rats. Cell Physiol Biochem 49: 172-189, 2018.

33. Potier M, Gonzalez JC, Motiani RK, Abdullaev IF, Bisaillon JM, Singer HA and Trebak M: Evidence for STIM1- and Orail-dependent store-operated calcium influx through ICRAC in vascular smooth muscle cells: Role in proliferation and migration. FASEB J 23: 2425-2437, 2009.

34. Earley S and Brayden JE: Transient receptor potential channels in the vasculature. Physiol Rev 95: 645-690, 2015.

35. Song S, Carr SG, McDermott KM, Rodriguez M, Babicheva A, Balistrieri A, Ayon RJ, Wang J, Makino A and Yuan JX: STIM2 (stromal interaction molecule 2)-mediated increase in resting cytosolic free $\mathrm{Ca}^{2+}$ concentration stimulates PASMC proliferation in pulmonary arterial hypertension. Hypertension 71: 518-529, 2018.

36. Kao SC, Wu H, Xie J, Chang CP, Ranish JA, Graef IA and Crabtree GR: Calcineurin/NFAT signaling is required for neuregulin-regulated Schwann cell differentiation. Science 323: 651-654, 2009.

37. Razmara M, Heldin $\mathrm{CH}$ and Lennartsson J: Platelet-derived growth factor-induced Akt phosphorylation requires mTOR/Rictor and phospholipase C- $\gamma 1$, whereas S6 phosphorylation depends on mTOR/Raptor and phospholipase D. Cell Commun Signal 11: 3, 2013.

38. Ogawa A, Firth AL, Smith KA, Maliakal MV and Yuan JX: PDGF enhances store-operated $\mathrm{Ca}^{2+}$ entry by upregulating STIM1/Orail via activation of Akt/mTOR in human pulmonary arterial smooth muscle cells. Am J Physiol Cell Physiol 302: C405-C411, 2012.

39. O'Callaghan DS, Savale L,Montani D, Jaïs X, Sitbon O, Simonneau G and Humbert M: Treatment of pulmonary arterial hypertension with targeted therapies. Nat Rev Cardiol 8: 526-538, 2011.

This work is licensed under a Creative Commons Attribution-NonCommercial-NoDerivatives 4.0 International (CC BY-NC-ND 4.0) License. 